\title{
Review: pharmacological and non-pharmacological interventions improve outcomes in patients with dementia and for their caregivers
}

\author{
Doody RS, Stevens JC, Beck C, et al. Practice parameter: management of dementia (an evidence-based review). Report of the \\ Quality Standards Subcommittee of the American Academy of Neurology. Neurology 2001 May 8;56:1154-66.
}

\section{QUESTION: Do pharmacotherapy, educational, or other non-pharmacological interventions improve outcomes in patients with dementia or for their caregivers?}

\section{Data sources}

Studies were identified by searching Medline, EMBASE/ Excerpta Medica, CINAHL, Current Contents, Psychological Abstracts, PsycINFO, and the Cochrane databases with terms that include Alzheimer's disease $(\mathrm{AD})$, vascular or multi-infarct dementia, dementia with associated parkinsonian disorder, progressive supranuclear palsy, frontotemporal dementia, and senile dementia. Additional terms used were question specific. Bibliographies of relevant papers were also reviewed.

\section{Study selection}

Studies were selected if they were randomised controlled trials published in any language or other types of studies published in English with $>20$ participants.

\section{Data extraction}

Data were extracted on study quality, participant characteristics, interventions, outcome measures, and results.

\section{Main results}

380 articles met the selection criteria. Several studies comparing cholinesterase inhibitors (eg, tacrine, donepezil, tartrate, and galantamine) with placebo showed that these drugs were more effective than placebo for improving cognitive outcomes in a subgroup of patients with mild-to-moderate $\mathrm{AD}$. Studies of cholinergic precursors (eg, lecithin) and muscarinic agonists (eg, xanomeline) for the treatment of $\mathrm{AD}$ have not shown beneficial effects. 1 large 2 year study showed that selegiline, $5 \mathrm{mg}$ taken orally twice daily, and vitamin E, $1000 \mathrm{IU}$ taken orally twice daily, delayed the time to a composite outcome indicator of clinical worsening of $\mathrm{AD}$ symptoms; however, no additive effects were seen from the combined use of selegiline and vitamin E. Data are lacking to support the use of other antioxidant or anti-inflammatory drugs, or other putative diseasemodifying drugs in the treatment of $\mathrm{AD}$. Antipsychotic drugs were effective for treating agitation or psychosis in patients with dementia in whom environmental manipulation failed, and antidepressants (eg, selected tricyclics, monoamine oxidase-B inhibitors, and selective serotonin reuptake inhibitors) were effective for treating depression in patients with dementia. Evidence from observational studies showed that educating family caregivers of patients with $\mathrm{AD}$ improved caregiver satisfaction and delayed time to patient's institutionalisation, and that educating staff in long-term care facilities about $\mathrm{AD}$ minimised the unnecessary use of antipsychotic drugs. Randomised trials have shown that behaviour modification, scheduled toileting, and prompted voiding reduced urinary incontinence in people with dementia. Graded assistance, skills practice, and positive reinforcement increased functional independence.

\section{Conclusions}

Cholinesterase inhibitors improve outcomes in some patients with Alzheimer's disease (AD). Antipsychotics may be effective for treating agitation, and antidepressants may be effective for treating depression in patients with dementia. Education for family caregivers of patients with $\mathrm{AD}$ may improve caregiver and patient outcomes. Such non-pharmacological interventions as behavioural modification are also effective.

\section{COMMENTARY} for future research. physicians have been published by a Canadian group. ${ }^{34}$ should be considered when choosing which drug to use. effects. them useful. 2001;(2):CD000442. 2001;(2):CD000464. [Editorial]. Can Med Assoc J 1991;144:851-3. Dementia. Can Med Assoc J 1999;160(12 Suppl):S1-15.
Source of funding: no
external funding.

For correspondence:

Quality Standards

Subcommittee,

American Academy of

Neurology, 1080

Montreal Avenue, St.

Paul, MN 55116, USA.

Fax +1713798

5326.

Abstract and

commentary also

appear in

Evidence-Based Mental

Health. A modified

version of this abstract

appears in

Evidence-Based

Nursing.

The guidelines by Doody et al provide an excellent review of the current literature on the use of pharmacological and non-pharmacological (including educational) interventions for the management of AD. Furthermore, the authors offer sensible recommendations

Doody et al used rigorous methods to complete a broad review of the literature on the management of dementia. Although the Cochrane databases were consulted, none of the relevant Cochrane reviews were cited (eg, the reviews on selegiline ${ }^{1}$ and thioridazine ${ }^{2}$ ). The authors suggest that the guideline is directed to neurologists and all other clinicians who manage dementia, but they do not state that it is targeted specifically to primary care physicians. Other guidelines targeted specifically to primary care

Although Doody et al offer guidelines for the use of the cholinesterase inhibitors (primarily donepezil, rivastigmine, and galantamine), they point out that because no head to head trials of these drugs exist they cannot recommend one over the others. Therefore, at present, the differences in dosing schedules and adverse effect profiles

In addition to excellent studies on the use of drug treatment in patients with $\mathrm{AD}$, good evidence exists in support of non-pharmacological interventions. Educational and non-pharmacological interventions are probably underused by many but may be as effective as a drug treatment and do not present the burdens of drug-related adverse

These guidelines provide an excellent summary of managing patients with dementia from a broad perspective, and clinicians from a variety of practice settings will find

A Mark Clarfield, MD Soroka Hospital Centre and Ben Gurion University of the Negev Beersheva, Israel

1 Birks J, Flicker L. Selegiline for Alzheimer's disease. Cochrane Database Syst Rev

2 Kirchner V, Kelly CA, Harvey RJ. Thioridazine for dementia. Cochrane Database Syst Rev

3 Clarfield AM, Bass MJ, Cohen C, et al. Assessing dementia: the Canadian Consensus

4 Patterson CJ, Gauthier S, Bergman H, et al. The recognition, assessment and management of dementing disorders: conclusions from the Canadian Consensus Conference on 